\title{
Introduction to the feature issue on design and evaluation of road network pricing
}

\author{
Theodore Tsekeris
}

Accepted: 8 October 2008 / Published online: 22 October 2008

(C) Springer Science + Business Media, LLC 2008

Several pricing strategies are increasingly considered worldwide as possible options to mitigate the negative externalities of traffic congestion in urban networks, manage travel demand and improve urban living conditions. Despite the annotated beneficial impacts of congestion pricing schemes on network performance and social welfare, a range of problems still hinder many decision makers to apply these schemes in practice. Such problems typically relate to technical issues as well as the use of toll revenues to address equity concerns and increase public acceptability. At the same time, the plans for upgrading, expanding or constructing new road transport infrastructure to enhance regional accessibility and economic development are hampered by limitations on the allocation of available public funds. This situation has motivated the development of suitable privatization mechanisms to allow the construction and operation of road infrastructure from profit-maximizing private firms, usually subject to constraints imposed by governments/regulators. The involvement of many different actors with diverse, often conflicting, goals and constraints, and complexities associated with realistic network implementation raise the need for devising innovative design and evaluation approaches for alternative road pricing schemes. This feature issue of Netnomics brings together original research, methods, state-of-the-art surveys and applications of pricing strategies in test and real-world road networks.

Tsekeris and $\operatorname{Vo} \beta$ [6] provides an in-depth extensive review of the state-ofthe-art and describes methodological advances in the design and evaluation of road pricing. It stresses the need for considering a number of paradigm shifts from the two polar cases of marginal social cost pricing of traffic congestion and revenue-maximizing (monopolistic or competitive) private road pricing in

\footnotetext{
T. Tsekeris $(\bowtie)$

Centre for Planning and Economic Research (KEPE), Athens, Greece

e-mail: tsek@kepe.gr
} 
order to handle a number of realistic design complexities and constraints. It also signifies the crucial role of the joint consideration of road pricing strategies with optimal capacity provision and several network management and regulatory measures. An integrated evaluation framework is finally suggested to allow incorporating a wide range of road pricing impacts into the overall scheme design process.

Balwani and Singh [1] investigates the implementation of distance-based road user charges in the urban area of Leeds, based on variations of values principally proposed in the U.K. National Transport Model (NTM). In this model, the urban areas are divided into rather concentric zones, indicating the inner city, suburbs, and fringe areas each having a uniform charge rate with the highest charge in the centre and lower subsequent charges as one moves out of the city. The paper suggests an alternative "medium charge" scenario, which provides a strategic hierarchy of and reduced difference between charges across the network. In this way, it can avoid significant traffic disruptions induced by the NTM-based charges due to extensive rerouting to the outer end of each concentric zone. The proposed scheme shows the potential to reduce traffic congestion and the number and average length of trips, and deliver positive net economic benefits and revenues, which may have implication for the future design of national (universal) road pricing schemes.

Yin and Lawphongpanich [7] addresses a common misconception in the literature regarding the case of 'first-best' marginal-cost pricing in road networks when not all utilized links are tolled. By using an algebraic proof as well as a numerical example, it shows that link-based Pigouvian tolls are sufficient but not generally necessary to reach a system-optimum traffic flow pattern. In particular, it suggests that system-optimal path-based tolls in an elastic demand setting satisfy the 'first-best' principle of marginal-cost pricing even if all links on the used paths are not tolled at Pigouvian levels or even tolled at all. As a result, such (valid) toll patterns can maximize social welfare, similar to charging the Pigouvian tax on every link, while they allow flexibility in the design of the road pricing scheme.

Extending previous work, Ekström et al. [4] handles the problem of finding the optimal number of toll facilities, their locations and individual toll levels, with the objective to maximize the net social surplus. The proposed continuous approximation procedure can potentially allow enhancing the efficiency and robustness of the toll design process, particularly in larger scale networks, compared to an existing heuristic. The results signify the importance of considering the collection (setup and operating) costs on the design and evaluation of second-best congestion tolling schemes.

Dimitriou and Tsekeris [2] describes an evolutionary game-theoretic model which represents the doubly dynamics of network traffic evolution in the hypothetical presence of dynamic congestion pricing in a real urban road network in Athens, Greece. Specifically, the model takes into account both the within-day dynamics of user responses to marginal cost pricing (MCP), under stochastic user equilibrium (SUE) conditions, and the day-to-day dynamics of travel demand. In order to enhance the realism of the evaluation process, it 
regards the route choice uncertainty and travel time reliability, together with the heterogeneity of the value of time and endogenously updated information acquisition rate of users. The results demonstrate the significance of regarding such dynamic and stochastic aspects of user responses on determining the beneficial impact of congestion charges on network performance.

Liu et al. [5] examines jointly the congestion pricing with revenue redistribution (refunding). These schemes are commonly considered in the literature as two separate, sequential steps; such a treatment may result in an inconsistency and instability in the solution of the traffic assignment procedure. The authors suggest a method and derive theoretical conditions of existence for the Pareto-improving and revenue-neutral link toll pricing in a two-mode (road and transit) network used by travelers with different value-of-time (VOT) distributions. Specifically, the proposed scheme can simultaneously determine appropriate levels of positive automobile user charges and negative transit user charges (subsidy) to achieve improvement of the system performance, make every individual user better off and have zero total revenue.

Dimitriou et al. [3] provides a number of modeling extensions to existing approaches dealing with the joint network design and pricing problem in monopolistic private highways competing with free urban roads. The profitmaximizing strategies of the system designer/operator take into account the spatial heterogeneity of toll prices among different highway access points (entry-based toll pricing) and origin-destination pairs (entry-exit-based toll pricing). The proposed approach incorporates the route choice uncertainty and represents the responses of users of multiple value-of-time groups, hence circumventing potential problems of underestimating the benefit of road pricing. A genetic algorithm is employed to address the problem complexity, which is increased due to the treatment of road capacity as a discrete variable. Real-world application results indicate that an appropriate combination of highway investment and entry-exit-based tolling scheme with level-of-service regulation can yield the largest gain in the net social benefit.

The papers contained in this feature issue can stimulate future research efforts to develop road network pricing schemes more responsive to the emerging policy needs, social concerns and market conditions. They can also help road providers/operators make more informed decisions which affect the users and the economy, through giving appropriate attention to the impact that different design parameters and behavioral aspects have on the evaluation of alternative road pricing strategies.

\section{References}

1. Balwani, A., \& Singh, S. (2009). Network impacts of distance-based road user charging. NETNOMICS: Economic Research and Electronic Networking, (this issue). doi:10.1007/ s11066-008-9025-y.

2. Dimitriou, L., \& Tsekeris, T. (2009). Evolutionary game-theoretic model for dynamic congestion pricing in multi-class traffic networks. NETNOMICS: Economic Research and Electronic Networking, (this issue). doi:10.1007/s11066-008-9027-9. 
3. Dimitriou, L., Tsekeris, T., \& Stathopoulos, A. (2009). Joint pricing and design of urban highways with spatial and user group heterogeneity. NETNOMICS: Economic Research and Electronic Networking, (this issue). doi:10.1007/s11066-008-9021-2.

4. Ekström, J., Engelson, L., \& Rydergren, C. (2009). Heuristic algorithms for a second-best congestion pricing problem. NETNOMICS: Economic Research and Electronic Networking, (this issue). doi:10.1007/s11066-008-9019-9.

5. Liu, Y., Guo, X., \& Yang, H. (2009) Pareto-improving and revenue-neutral congestion pricing schemes in two-mode traffic networks. NETNOMICS: Economic Research and Electronic Networking, (this issue). doi:10.1007/s11066-008-9018-x.

6. Tsekeris, T, \& Vo $\beta$, S. (2009). Design and evaluation of road pricing: state-of-the-art and methodological advances. NETNOMICS: Economic Research and Electronic Networking, (this issue). doi:10.1007/s11066-008-9024-z.

7. Yin, Y., \& Lawphongpanich, S. (2009). Alternative marginal-cost pricing for road networks. NETNOMICS: Economic Research and Electronic Networking, (this issue). doi:10.1007/ s11066-008-9020-3. 